\title{
Implementación de un voltímetro digital con voz incorporada
}

\begin{abstract}
RESUMEN
El presente artículo describe el resultado de un estudio de investigación realizada en el Instituto de Investigación de la Facultad de Ingeniería Electrónica y Eléctrica de la UNMSM, como consecuencia del objetivo planteado se ha llegado a implementar un voltímetro digital $\mathrm{DC}$ del cual el usuario del voltímetro puede escuchar a través de un parlante una voz que le informa la cantidad de voltios que el voltímetro está midiendo. La metodología empleada para ello se basa en la digitalización de la voz que se almacena en una memoria EEPROM serial, un microcontrolador gobierna el proceso. Adicionalmente el valor de la medida también puede ser leída a través de un display LCD.
\end{abstract}

Palabras claves: Voltímetro Digital DC, voz, microcontrolador, EEPROM, DAC

IMPLEMENTATION OF A DIGITAL VOLTMETER WITH INTEGRATED VOICE

\section{ABSTRACT}

This article describes the results of a research study conducted at the Institute for Research of the Faculty of Electronic and Electrical Engineering of the UNMSM. Following the focus of the study was implemented a digital voltmeter DC, which issues through a loudspeaker a voice that informs the user of the amount of volts that the voltmeter is measuring. The methodology is based on the digitization of the voice that is stored in a serial EEPROM memory, microcontroller governs the process. Additionally, the measurement value can also be read through an LCD display.

Keywords: DC Digital Voltmeter, voice, microcontroller, EEPROM, DAC

\section{INTRODUCCIÓN}

Un voltímetro DC (Direct Current) es un instrumento electrónico que se utiliza para medir la diferencia de potencial entre dos puntos de un circuito eléctrico DC [1]. Es utilizado por ingenieros, técnicos electrónicos, eléctricos así como también por aficionados.

El instrumento presentado es un prototipo que no tiene antecedentes similares, transforma el valor de la medida realizada a voz, la utilidad de poder escuchar el valor de la medida facilita el trabajo del usuario ya que él no tendrá que distraer su vista del objeto medido al observar un display, sino en cambio solo tendrá que escuchar el resultado de la medición. Esto es importante para la comodidad del usuario y para evitar que por distracción pueda producirse cortos circuitos, accidentes de otro tipo o medidas incorrectas.

El circuito puede ser extendido para medir otras magnitudes eléctricas tales como: voltios $A C$, amperios $A C$, amperios $D C$ y ohmios.

El circuito puede tener diversas aplicaciones debido a que cualquier evento del mundo real puede ser traducido a un voltaje proporcional DC mediante transductores especiales, luego según la aplicación que se trate ese voltaje puede ser asociado con múltiples mensajes de voces que se almacenan digitalmente en la memoria EEPROM (Electrically Erasable Programmable Read Only Memory).

El circuito ha sido implementado con un microcontrolador, una memoria serial EEPROM, un conversor digital analógico (DAC), un display LCD, un parlante, entre otros dispositivos.

Adicionalmente, a la voz se ha incorporado un display LCD en donde también se pueda leer el valor de la medida realizada.

\section{DISEÑO EXPERIMENTAL}

\section{Descripción del hardware}

El circuito es mostrado en la figura 1, consta de un microcontrolador 16F877A [2], memoria externa EEPROM 24LC512 de comunicación serial que utiliza dos pines para transferir el contenido de datos [3], conversor digital analógico DAC 0800 [4],

\footnotetext{
Master en Ing. Electrónica - USP, Brasil. Ing. Electrónico - UNMSM, Perú. Profesor Principal. Director del Instituto de Investigaciones de la FIEE.
}

E-mail: guillermotm@hotmail.com 
amplificador de audio LM386 [5], un parlante por donde se escucha el valor de la medida realizada, oscilador y display LCD por donde también es leída numéricamente el valor de la medida.

El circuito no está limitado por la capacidad interna de la memoria del microcontrolador debido a que utiliza memoria externa EEPROM de comunicación serial de sólo 4 pines, en ella se han grabado previamente las voces digitalizadas.

Cuando mediante el microcontrolador se lee o extrae información de la memoria se hace respetando el protocolo de comunicación serial I2C (Inter-Integrated Circuit), cada palabra de la memoria es leída a intervalos de 125 useg.
El microcontrolador incorpora internamente cuatro conversores analógicos digitales, uno de los cuales tiene su entrada por el pin A4 del microcontrolador. Es por este pin, que ingresa el voltaje medido, valor con el que el programa asocia un sector particular de la memoria EEPROM. A partir de este momento, se extrae todas las palabras digitales de ese sector que corresponden a la voz digitalizada del valor medido.

Luego, de extraer de la memoria cada byte de la voz digitalizada, éstos son enviados desde el microcontrolador hacia el DAC0800 para su conversión en un valor analógico, que luego de ser amplificado mediante el LM386 excita al parlante para su conversión en voz.

Figura 1. Circuito Lógico del prototipo

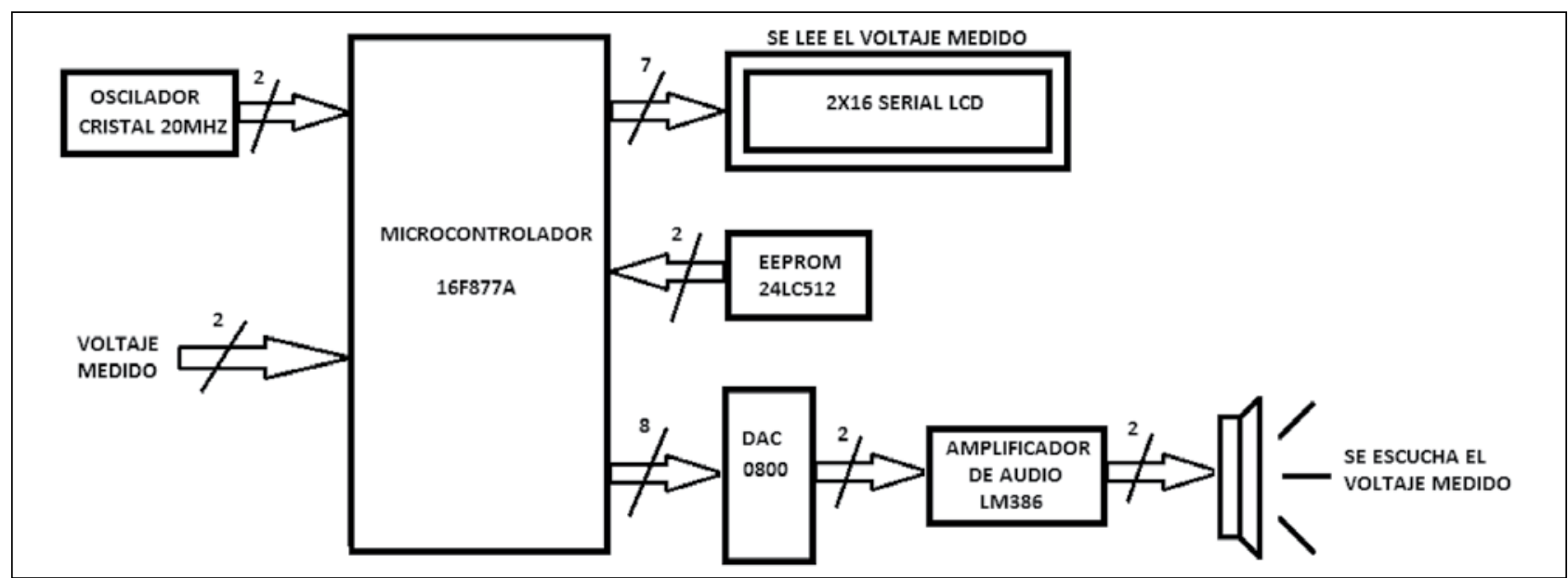

Fuente: Elaboración propia

Figura 2. Secuencia de ejecución del programa

\section{Descripción del Software}

La figura 2, muestra la secuencia de ejecución del programa que ha sido escrito en lenguaje $\mathrm{C}++$ [6]. El programa se ejecuta apenas ingresa el valor medido de voltaje, su valor opcionalmente es presentado en un display LCD, cada valor que es medido está asociado con un sector de la memoria equivalente en voz de esa medida, de las direcciones correspondientes de ese sector son extraídos bit por bit de cada byte de la voz digitalizada respetando el protocolo I2C de comunicación que se establece entre el microcontrolador con la memoria EEPROM.

Cada byte de la voz, luego de ser extraída de la memoria, se envía cada $125 \mathrm{us}(8 \mathrm{KHz})$ al DAC, para ello se ha tenido que agregar retardos por software. Esto es necesario porque la voz se digitalizó a una frecuencia de muestreo de $8 \mathrm{Khz}$ por lo que su reproducción también tiene que hacerse a esa frecuencia.

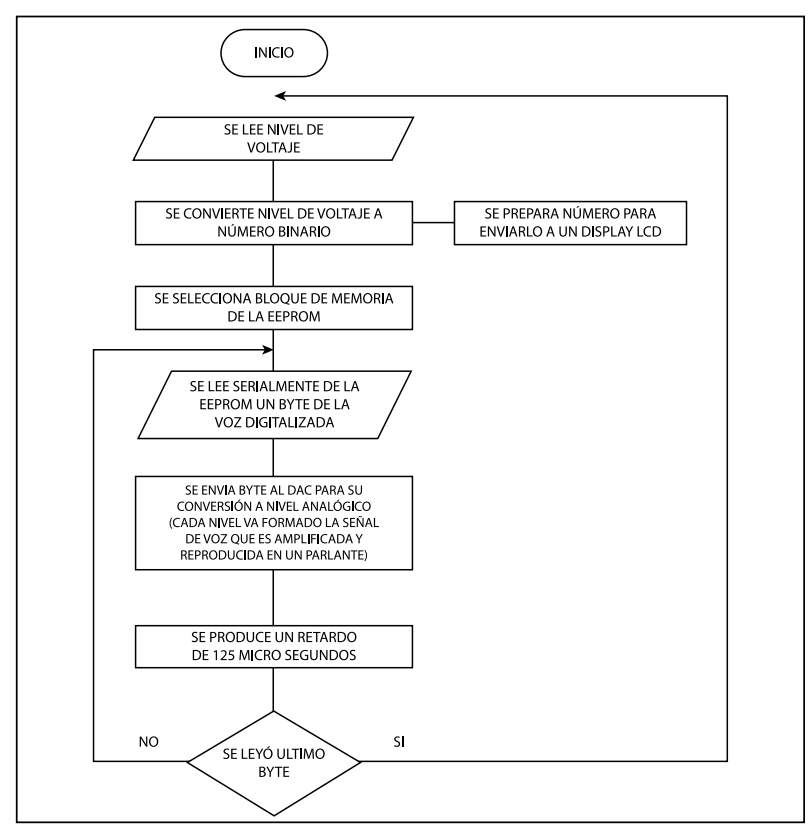

Fuente: Elaboración propia 


\section{METODOLOGÍA}

El programa residente en el PIC ha sido realizado en lenguaje $\mathrm{C}$ con el compilador PCW.

La voz digitalizada en la memoria EEPROM han sido previamente grabadas en formato WAV con el grabador de sonidos de Windows XP a 8Khz. Posteriormente con la herramienta HEXEDIT el archivo WAV ha sido convertido a un formato hexadecimal que posteriormente se ha grabado en la memoria EEPROM.

Para editar la voz se ha utilizado el utilitario Power Sound Editor Free, tanto ese utilitario, como el HEXEDIT y el PCW se han instalado en una LABTOP Lenovo, Core i3, $2.53 \mathrm{GHz}$, de 4GB de RAM. Para grabar el PIC 16F877A y la EEPROM 24LC512 se ha utilizado el Programador DPIC conectado por USB al LABTOP.

\section{RESULTADOS}

Las medidas convertidas en voces han sido validadas con las leídas en un multímetro Fluke modelo 175.

La foto de la figura 3 muestra el circuito implementado, el cual ha sido montado sobre un protoboard, también se puede apreciar la caja del parlante utilizado.

Figura 3. Fotografía del prototipo

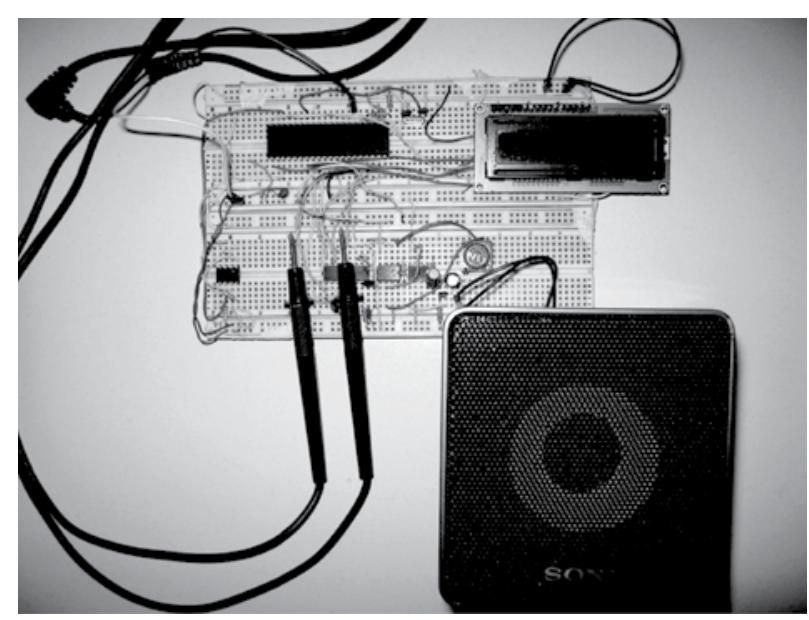

Fuente: Elaboración propia

\section{CONCLUSIONES}

1. Se ha implementado un prototipo de voltímetro DC que reproduce el valor medido mediante voz, que previamente se ha digitalizado y almacenado en una memoria EEPROM. Adicionalmente, el valor medido se muestra en un display LCD.

2. El prototipo implementado reproduce a voz, niveles de voltaje medidos de 0 a 5 voltios DC, pero puede ser extendido para mayores voltajes e inclusive para medidas de voltajes AC. Para ello, se tendrá que emplear mayores recursos de memoria, sin variaciones complicadas en el programa.

3. El conocimiento obtenido puede ser adaptado para otras aplicaciones en donde se desee convertir una variable eléctrica a voz.

\section{REFERENCIAS BIBLIOGRÁFICAS}

[1] Boylestad R. (2004). Introducción al Análisis de Circuitos. Pearson, Prentice Hall, México.

[2] MICROCHIP (2003). PIC16F87XA Data Sheet 28/40/44-Pin Enhanced Flash Microcontrollers. http://ww1.microchip.com/downloads/ en/DeviceDoc/39582b.pdf (Visitado el 02-022011).

[3] MICROCHIP (2008). EEPROM 24LC512512K I2C ${ }^{\text {TM }}$ CMOS Serial EEPROM. http:// ww1.microchip.com/downloads/en/ DeviceDoc/21754J.pdf ( Visitado 02-02-2011).

[4] National Semiconductor (2006). DAC0800/ DAC0802 8-Bit Digital-to-Analog Converters. http://www.ti.com/lit/ds/symlink/dac0800.pdf (Visitado el 02-02-2011).

[5] National Semiconductor (2000). LM386 Low Voltage Audio Power Amplifier. http://www. ti.com/lit/ds/snas545a/snas545a.pdf (Visitado 02-02-2011).

[6] Peñalosa E. (2004). Fundamentos de Programación C/C++. Alfaomega Grupo Editor, México. 